\title{
Hepatitis B and Hepatocellular Carcinoma Screening Among Asian Americans: Survey of Safety Net Healthcare Providers
}

\author{
Mandana Khalili $\cdot$ Jennifer Guy $\cdot$ Albert Yu • \\ Alexander Li $\cdot$ Nadia Diamond-Smith • \\ Susan Stewart • Moon Chen Jr. • Tung Nguyen
}

Received: 11 June 2010/ Accepted: 19 September 2010/Published online: 3 November 2010

(c) The Author(s) 2010. This article is published with open access at Springerlink.com

\begin{abstract}
Background Physician patterns of screening for hepatitis B (HBV) and hepatocellular carcinoma (HCC) among Asian Americans are not well described.

Aims To describe HBV and HCC screening practices among providers with large Asian American populations.

Methods Providers within San Francisco's safety net system were surveyed with respect to HBV and HCC screening practices as well as knowledge, attitudes, and barriers to HCC screening.

Results Among the 109 respondents (response rate $=$ $72 \%$ ), $62 \%$ were aged $>40,65 \%$ female, $24 \%$ Asian, $87 \%$ primary care providers, and $48 \%$ had $>25 \%$ Asian patients. Only $76 \%$ had screened $>50 \%$ of their Asian patients for
\end{abstract}

M. Khalili $(\bowtie) \cdot$ J. Guy $\cdot$ N. Diamond-Smith

Department of Medicine, University of California San Francisco,

San Francisco General Hospital, 1001 Potrero Ave, NH-3D,

San Francisco, CA 94110, USA

e-mail: mandana.khalili@ucsf.edu

A. $\mathrm{Yu}$

Department of Family and Community Medicine, University

of California San Francisco, San Francisco, CA, USA

A. $\mathrm{Yu}$

Chinatown Public Health Center, San Francisco Department of Public Health, San Francisco, CA, USA

\author{
A. Li \\ LA Care Health Plan, Los Angeles, CA, USA \\ S. Stewart · T. Nguyen \\ Department of Medicine, University of San Francisco, \\ San Francisco, CA, USA \\ M. Chen Jr. \\ Department of Internal Medicine, University of California Davis, \\ Sacramento, CA, USA
}

HBV and $43 \%$ had vaccinated $>50 \%$ of eligible patients against HBV. Although 94\% knew Asians were disproportionately affected by HCC, only $79 \%$ had screened for HCC in $>50 \%$ of their Asian patients with chronic hepatitis B (CHB). A majority believed that HCC screening in CHB reduces HCC mortality (70\%) and is cost-effective (57\%). The most common HCC screening modality was AFP with abdominal ultrasound every 6-12 months (63\%). Factors associated with HBV screening were familiarity with AASLD guidelines (OR 6.4, 95\% CI 1.3-30.1, $p=0.02$ ) and having vaccinated $>50 \%$ of eligible patients against HBV (OR 2.2, 95\% CI 1.1-4.5, $p=0.03$ ). Factors associated with HCC screening using abdominal ultrasound every 6-12 months were having $>25 \%$ Asian patients $(\mathrm{OR}=4.5$, 95\% CI 1.3-15.3, $p=0.02$ ) and higher HCC knowledge score $(\mathrm{OR}=1.9$ per item, 95\% CI 1.01-3.6, $p=0.045)$. Conclusions $\mathrm{HBV}$ and $\mathrm{HCC}$ screening rates and $\mathrm{HBV}$ vaccination among Asians from physician report is suboptimal. HCC screening is associated with having more Asian patients and higher provider knowledge. Provider education is essential in increasing rates of $\mathrm{HBV}$ and HCC screening among Asian Americans.

Keywords Hepatocellular carcinoma surveillance . Hepatitis B screening · Asian American · Provider practices

$\begin{array}{ll}\text { Abbreviations } \\ \text { HCC } & \text { Hepatocellular carcinoma } \\ \text { HBV } & \text { Hepatitis B virus } \\ \text { CHB } & \text { Chronic hepatitis B infection } \\ \text { HBsAg } & \text { Hepatitis B surface antigen } \\ \text { Anti-HBs } & \text { Hepatitis B surface antibody } \\ \text { Anti-HBc } & \text { Hepatitis B core antibody } \\ \text { US } & \text { Ultrasound }\end{array}$


CT Computerized tomography

AFP Alpha fetoprotein

AASLD American Association for the Study of Liver Diseases

CDC Centers for Disease Control and Prevention

\section{Introduction}

Approximately 1.25 million Americans have chronic hepatitis B infection (CHB). Patients with CHB are at significant lifetime risk for developing cirrhosis, liver failure, and hepatocellular carcinoma (HCC) [1]. CHB is especially prevalent among Asians and these patients have a threefold or greater increase in the rate of HCC as compared to nonHispanic Whites [2-4]. The prevalence of CHB varies nationwide due largely to immigration patterns from highrisk areas. In the San Francisco Bay Area, where onefourth of the population is from the highly endemic regions of Asia and the Pacific Islands, the reported prevalence of $\mathrm{CHB}$ is as high as 9\% among these individuals [5]. Moreover, in 2006 the San Francisco Department of Public Health reported that $84 \%$ of probable cases of HBV infection occurred in the Asian American and Pacific Islander (AAPI) populations [6]. Thirty percent of the patients in the San Francisco's safety net healthcare system are AAPIs [7]. Most of the patients in this system are uninsured or underinsured and have significant health care disparities $[8,9]$. In order to reduce the burden of HCC among AAPIs, it is important that these patients are screened for HBV and, depending on their status, receive HBV vaccination or HCC screening as appropriate, especially within the safety net healthcare systems [10].

Hepatocellular carcinoma screening can detect early stage disease and may improve survival in the presence of curative treatment options [11]. Practice guidelines published by the American Association for the Study of Liver Diseases (AASLD) recommend HCC screening in high-risk populations, including Asians with CHB [12]. The recommended screening test is liver ultrasound every 6-12 months. Studies have shown that the majority of providers recommend HCC screening to their patients [13, 14], however, there is no information on the screening modalities typically employed by providers within the context of CHB infection. In addition, although there is limited data on provider knowledge, attitudes, and perceived barriers to HCC surveillance among HBV-infected Asian Americans [14, 15], there are no studies evaluating these factors within the resource-limited safety net healthcare systems. This information is crucial in identifying interventions to reduce disparities in health and health care in this at-risk population.

In this study we aimed to characterize provider-reported screening practices for $\mathrm{HBV}$ and $\mathrm{HCC}$ along with provider knowledge, attitudes, and perceived barriers to $\mathrm{HCC}$ screening among Asian Americans by surveying a large network of providers within San Francisco's safety net healthcare system.

\section{Methods}

\section{Study Design}

This was a cross-sectional survey of primary care and specialty providers who provide health care in clinics affiliated with the San Francisco's safety net healthcare system, which annually provides care for over 150,000 patients, including most of the county's uninsured residents [7]. This system is composed of the San Francisco Community Clinic Consortium, which has ten nonprofit primary care community health centers, and the San Francisco Department of Public Health's Community Health Network, which includes 11 community-based primary care clinics and one acute care hospital (San Francisco General Hospital) with on-site primary and specialty care clinics [7]. The survey was sent to the 150 providers from these primary care clinics and three specialty (gastroenterology, hepatology, HIV/infectious diseases) clinics by mail or electronic mail between December 1, 2007, and March 31, 2008. A second mailing to non-respondents was conducted after 4 weeks. Respondents chose a $\$ 10$ gift certificate or a chance for a $\$ 100$ gift card as incentives. The study was approved by the Committee on Human Research of the University of California, San Francisco, and the SFGH Data Governance Committee.

\section{Survey Design}

The survey instrument was developed by the study investigators with input from hepatology expert opinion and previously published surveys. Content domains included provider and practice characteristics, HBV screening and CHB management practices, HCC surveillance practices and modalities employed, provider knowledge of $\mathrm{HBV}$ and $\mathrm{HCC}$, and attitudes toward (and perceived barriers to) HCC surveillance. The survey was pilot-tested with 20 physicians and revised.

\section{Data Definitions and Statistics}

Provider and practice characteristics were summarized using mean $\pm \mathrm{SD}$, median (range), and frequencies. In order to assess overall provider knowledge, favorable attitudes, and barriers to performing HCC surveillance, scores were formed from the responses to questions assessing these factors. The knowledge score was 
computed as the number of correct responses to the four questions assessing knowledge (minimum 0, maximum 4). The attitude score was determined by summing the numerical codes assigned to responses to eight questions assessing provider attitudes as follows: 1 to an "agree" response, 0.5 to "unsure" response, and " 0 " to a "disagree" response. The barrier score was computed as the number of barriers from a list of 11 categories including an "other" category identified by the providers. Screening for HCC was defined as performing ultrasound every 6-12 months. To evaluate provider and practice factors associated with $\mathrm{HBV}$ and $\mathrm{HCC}$ screening, univariate analysis was performed using Student's $t$ test for continuous variables and Chi-square (Fishers exact test when appropriate) tests for categorical variables. Multivariable logistic regression modeling evaluating factors associated with HBV and HCC screening included independent predictors from an a priori compiled list as well as those with a $p$ value $\leq 0.2$ as determined by the univariate analysis. Statistical significance was assessed at the $p$ value of $<0.05$ level (two-sided) in all models. All analyses were performed using STATA version 8.0 (STATA Corporation, College Station, TX).

\section{Results}

Provider and Practice Characteristics

Out of 150 providers, 109 returned the survey, a response rate of $72 \%$. Provider characteristics are shown in Table 1. Most $(87 \%)$ were primary care providers, 9\% were hepatologists and gastroenterologists, and $80 \%$ held an MD degree. The majority were above age 40 and $65 \%$ were female. Thirty-one percent were Asian and half had been in practice for more than 10 years. Twenty-four percent of all providers and $77 \%$ of the Asian providers spoke an Asian language. About half (48\%) reported that Asian patients comprised over $25 \%$ of their patient population and $53 \%$ reported that among their Asian patients, more than $50 \%$ had limited English proficiency. The majority of patients in the practices surveyed were either uninsured or had public insurance.

\section{HBV Screening, Vaccination, and HCC Screening}

Provider management practices relating to HBV screening, HBV vaccination, and HCC surveillance among Asian patients are summarized in Table 2 . Only $76 \%$ of the providers reported that $>50 \%$ of their Asians patients had been screened for HBV. Only $43 \%$ of the providers reported that $>50 \%$ of their eligible patients had received $\mathrm{HBV}$ vaccination. A multitude of $\mathrm{HBV}$ screening practices
Table 1 Provider and practice characteristics in the San Francisco safety net healthcare system

\begin{tabular}{|c|c|}
\hline \multicolumn{2}{|l|}{ Age $(\%)$ (years) } \\
\hline $20-29$ & 6 \\
\hline $30-39$ & 29 \\
\hline $40-49$ & 28 \\
\hline $50-59$ & 28 \\
\hline$\geq 60$ & 9 \\
\hline Female $(\%)$ & 65 \\
\hline \multicolumn{2}{|l|}{ Race/ethnicity (\%) } \\
\hline Caucasian & 61 \\
\hline African-American & 1 \\
\hline Asian/Pacific Islander & 31 \\
\hline Latino & 6 \\
\hline Other & 1 \\
\hline Born in United States (\%) & 85 \\
\hline Speak Asian language (\%) & 24 \\
\hline \multicolumn{2}{|l|}{ Post-graduate degree $(\%)$} \\
\hline MD & 80 \\
\hline Nurse practitioner & 17 \\
\hline Other & 3 \\
\hline \multicolumn{2}{|l|}{ Specialty $(\%)$} \\
\hline Internal medicine & 46 \\
\hline Family practice & 41 \\
\hline Gastroenterology/hepatology & 9 \\
\hline Infectious disease & 2 \\
\hline Other & 2 \\
\hline \multicolumn{2}{|l|}{ Years in practice $(\%)$} \\
\hline $0-10$ & 50 \\
\hline $11-20$ & 43 \\
\hline$>20$ & 7 \\
\hline Median number of patients seen per week (range) & $30(5-100)$ \\
\hline More than $25 \%$ Asian patients & 48 \\
\hline $\begin{array}{l}\text { More than } 50 \% \text { of Asian patients with limited English } \\
\text { proficiency }\end{array}$ & 53 \\
\hline \multicolumn{2}{|l|}{ Median proportion of patients in practice with } \\
\hline Private insurance & 5 \\
\hline Public insurance & 50 \\
\hline Uninsured & 40 \\
\hline
\end{tabular}

$(n=109)$

were reported: $48 \%$ ordered $\mathrm{HBsAg}$ only; $16 \%$ used HBsAg and anti-HBs in combination; 24\% used HBsAg in combination with anti-HBs and total anti-HBc; $9 \%$ used HBsAg and total anti-HBc; 2\% used anti-HBc; and $1 \%$ used HBV viral load.

There was also considerable variation in defining highrisk populations for HCC screening-60\% would screen all patients with $\mathrm{CHB}, 11 \%$ would screen Asian patients with CHB, $6 \%$ would screen only in patients with HBV-induced cirrhosis, and $23 \%$ would screen a combination of these 
Table 2 HBV screening, vaccination, and management among San Francisco safety net providers

\begin{tabular}{|c|c|}
\hline Screening and management & Proportion $(\%)$ \\
\hline \multicolumn{2}{|c|}{ Proportion of Asian patients that have been screened for HBV } \\
\hline$\leq 25 \%$ & 10 \\
\hline $26-50 \%$ & 11 \\
\hline $51-74 \%$ & 26 \\
\hline$\geq 75 \%$ & 50 \\
\hline Not sure & 3 \\
\hline \multicolumn{2}{|c|}{ Proportion of eligible patients that have been vaccinated against HBV } \\
\hline$\leq 25 \%$ & 25 \\
\hline $26-50 \%$ & 21 \\
\hline $51-74 \%$ & 26 \\
\hline$\geq 75 \%$ & 17 \\
\hline Not sure & 11 \\
\hline \multicolumn{2}{|c|}{$\begin{array}{l}\text { Proportion of Asian patients with chronic HBV that have been } \\
\text { vaccinated against HAV }\end{array}$} \\
\hline$\leq 25 \%$ & 17 \\
\hline $26-50 \%$ & 10 \\
\hline $51-74 \%$ & 11 \\
\hline$\geq 75 \%$ & 50 \\
\hline Not sure & 12 \\
\hline \multicolumn{2}{|c|}{$\begin{array}{l}\text { Proportion of Asian patients with chronic HBV that have been } \\
\text { screened for HCC }\end{array}$} \\
\hline$\leq 25 \%$ & 4 \\
\hline $26-50 \%$ & 10 \\
\hline $51-74 \%$ & 13 \\
\hline$\geq 75 \%$ & 66 \\
\hline Not sure & 7 \\
\hline Offers therapy for HBV & 35 \\
\hline
\end{tabular}

$H A V$ hepatitis A virus, $H B V$ hepatitis B virus, $H C C$ hepatocellular carcinoma

groups. Only $79 \%$ reported that they had screened for HCC in $>50 \%$ of their Asian patients with CHB. Although 27\% of providers were unfamiliar with the AASLD guidelines for HCC screening in HBV patients, 94\% reported that they perform HCC screening among CHB patients.

\section{HCC Screening Modalities}

Providers were asked to report which of several laboratory or imaging studies they used for HCC screening, either alone or in combination. Overall, $91 \%$ used alpha fetoprotein (AFP); 79\% used ultrasound (US); $71 \%$ used AST and/or ALT; $46 \%$ used other imaging modalities; and 30\% used HBV viral load (Fig. 1). The most commonly used modalities reported were AFP (90\%) and US (78\%) at a frequency of every 6-12 month. Among the $88 \%$ who indicated that they used a combination of AFP and an imaging study for HCC surveillance every 6-12 months,

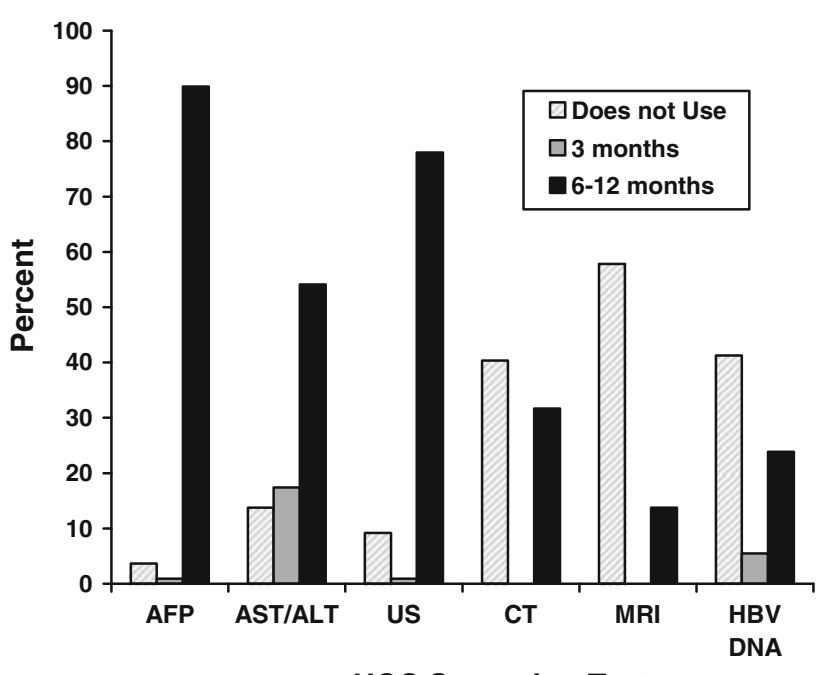

Fig. 1 Hepatocellular carcinoma screening modalities utilized by providers. The providers were asked whether they used any of the following laboratory and abdominal imaging modalities as a screening tool for hepatocellular carcinoma. The figure summarizes the responses of providers with regards to the type of test and the frequency of the test used in their practice for hepatocellular carcinoma surveillance. $A F P$ alpha fetoprotein, $A S T$ aspartate aminotransferase, $A L T$ alanine aminotransferase, $U S$ ultrasound, $C T$ computed tomography, MRI magnetic resonance imaging, $H B V D N A$ hepatitis B viral load

$63 \%$ used US, $8 \%$ used computed tomography (CT), and $29 \%$ used more than one type of imaging. Only $3 \%$ of respondents used AFP alone and 5\% used imaging alone for HCC surveillance.

Provider Knowledge, Attitudes, and Barriers to HCC Screening

Knowledge, attitudes, and barriers to HCC screening are summarized in Table 3. Nearly all providers knew that $\mathrm{CHB}$ was the most common cause of HCC among Asians and that Asians had a higher incidence of HCC compared to Whites. Three-quarters indicated that treating $\mathrm{CHB}$ can prevent HCC. About $70 \%$ believed that HCC screening in patients with $\mathrm{CHB}$ reduces HCC mortality and over half thought that screening is cost-effective and not screening poses a malpractice risk. The most common factors that influence a provider's decision to order any cancerscreening test were recommendations by a national organization (93\%) and good evidence that screening decreases mortality (99\%). The most common perceived barriers influencing a provider's decision to perform HCC screening among patients with $\mathrm{CHB}$ were lack of imaging resources (59\%), unclear HCC screening guidelines (35\%), difficulty accessing specialty care (35\%), and patient financial barriers $(31 \%)$. 
Table 3 Provider knowledge, attitudes, and barriers towards hepatocellular carcinoma (HCC) surveillance

\begin{tabular}{|c|c|}
\hline Knowledge (mean score $3.3 \pm 0.8$ ) & Correct answers $(\%)$ \\
\hline Asians have same incidence of liver cancer as Whites & 94 \\
\hline Most common cause of liver cancer in Asians is HBV & 95 \\
\hline Treating HBV can prevent liver cancer & 77 \\
\hline HCC screening in HBV reduces liver cancer mortality & 70 \\
\hline Attitude (mean score $=5.8 \pm 1.6$ ) & Percent agree \\
\hline Not screening for HCC is a malpractice risk & 59 \\
\hline Screening for HCC is cost-effective & 57 \\
\hline Will perform cancer screening when it is recommended by a national organization & 93 \\
\hline Will perform cancer screening when my patients ask for it & 69 \\
\hline Will perform cancer screening when it is used as a quality measure at my institution or by insurance companies & 72 \\
\hline Will perform cancer screening when it is covered by health insurance & 62 \\
\hline Will perform cancer screening when I see an increasing frequency of cancer in my practice & 56 \\
\hline Will perform cancer screening when there is good evidence that screening leads to decreased mortality & 99 \\
\hline Provider perceived barriers (mean score $=2.3 \pm 1.7$ ) & Percent agree \\
\hline Lack of clarity of liver cancer-screening guidelines & 35 \\
\hline Discomfort with discussing liver cancer screening & 1 \\
\hline Lack of effectiveness of cancer screening & 12 \\
\hline Lack of US/CT/MRI screening resources & 59 \\
\hline Lack of effective therapy for liver cancer & 15 \\
\hline Difficulty accessing specialty (GI/hepatology) care & 35 \\
\hline Lack of blood testing resources & 4 \\
\hline Language access barriers with patient & 18 \\
\hline Patient financial barriers & 31 \\
\hline Uncertain/unaware of liver cancer screening guidelines & 27 \\
\hline
\end{tabular}

$H B V$ hepatitis B virus, $H C C$ hepatocellular carcinoma

Table 4 Multivariate regression analysis of factors associated with hepatocellular carcinoma screening with abdominal ultrasound every 6-12 months

\begin{tabular}{llcl}
\hline Variable & Odds ratio & 95\% CI & $p$ value \\
\hline Age (per decade) & 0.7 & $0.3-1.7$ & 0.5 \\
Female gender & 0.5 & $0.1-1.6$ & 0.2 \\
Provider race (Asian vs. non-Asian) & 0.4 & $0.1-1.6$ & 0.2 \\
More than 10 years in practice & 1.3 & $0.3-6.7$ & 0.3 \\
Specialty (primary care vs. GI/hepatology) & 0.2 & $0.03-1.1$ & 0.06 \\
Familiarity with AASLD practice guidelines on HCC screening & 1.9 & $0.5-6.5$ & 1.0 \\
More than 25\% Asian patients in practice & 4.5 & $1.3-15.3$ & 0.02 \\
Provider knowledge score & 1.9 & $1.01-3.6$ & 0.045 \\
Provider attitude score & 1.1 & $0.8-1.6$ & 0.5 \\
Provider barrier score & 1.01 & $0.7-1.4$ & 0.06 \\
\hline
\end{tabular}

Factors Associated with Performing the Recommended HBV and HCC Screening

Factors associated with the higher likelihood of reporting HBV screening among $>50 \%$ of Asian patients in univariate analysis included having performed $\mathrm{HBV}$ vaccination in $>50 \%$ of eligible patients (94 vs. $60 \%, p<0.0001$ ),
HCC screening in $>50 \%$ of CHB patients in practice ( 86 vs. $43 \%, p<0.0001)$, familiarity with AASLD guidelines (84 vs. 52\%, $p=0.001$ ), and provider age less than 40 years ( 88 vs. $68 \%, p=0.02$ ). The rate of $\mathrm{HBV}$ screening (in more than $50 \%$ of their Asian patients) was not significantly different among those providers who spoke an Asian language versus those who did not speak an 
Asian language (88 vs. $70 \%, p=0.07)$. In addition, although a higher rate of HBV screening was reported by providers at safety net health centers with a high proportion of Asian providers who speak an Asian language, this did not reach statistical significance ( 90 vs. $72 \%, p=0.07$ ). On multivariable analysis when controlling for provider age, gender, race, years in practice, and specialty, familiarity with AASLD guidelines (OR 6.4, 95\% CI 1.3-30.1, $p=0.02$ ) and having vaccinated against $\mathrm{HBV}$ in $>50 \%$ of eligible patients (OR 2.2, 95\% CI 1.1-4.5, $p=0.03$ ) were significantly associated with a higher likelihood of reporting HBV screening among $>50 \%$ of Asian patients.

For HCC screening, in univariate analysis, performing US every 6-12 months was associated with having $>25 \%$ or more Asian patients [53\% (45/85) vs. $25 \%$ (6/24), $p=0.02]$ and higher knowledge score $(3.0 \pm 0.9$ vs. $3.4 \pm 0.8, p=0.01$ ). There was no statistically significant difference in performing US every 6-12 months among providers who spoke an Asian language compared to those who did not speak an Asian language (88 vs. $75 \%$, $p=0.2$ ) and among providers from centers with a high proportion of Asian providers who spoke an Asian language compared to those who did not practice at these centers ( 90 vs. $75 \%, p=0.1$ ). In multivariate analysis after controlling for provider age, gender, race, years in practice, and medical specialty the same two variables, having $>25 \%$ of Asian patients in the practice $(\mathrm{OR}=4.5,95 \% \mathrm{CI}$ $1.3-15.3, p=0.02$ ) and having a higher knowledge score $(\mathrm{OR}=1.9,95 \%$ CI $1.01-3.6, p=0.045)$, remained significantly associated with performing ultrasound every 6-12 months (Table 4).

\section{Discussion}

This is the first study to evaluate provider-reported HBV and HCC screening practices as well as knowledge, attitudes, and barriers towards HCC screening among Asian Americans in a safety net healthcare system. Respondents reported suboptimal rates of screening for and vaccination against HBV among Asians and screening for HCC. Having a higher rate of $\mathrm{HBV}$ vaccination in practice and familiarity with AASLD guidelines was associated with higher rates of HBV screening whereas having a large proportion of at-risk Asian patients and greater provider knowledge of HCC's relationship to HBV were associated with HCC screening.

At the time of this survey, the Center for Disease Control and Prevention (CDC) recommended HBV screening in Asian and Pacific Islanders and vaccination of all eligible at-risk patients. The current HBV screening recommendations also include US born persons not vaccinated as infants whose parents were born in regions with high HBV endemicity [16]. The recent Institute of Medicine Hepatitis and Liver Cancer Report also emphasizes the importance of HBV screening and vaccination [17]. Studies to date have shown that HBV screening and vaccination among Asian Americans are suboptimal at rates of 35-65\% $[15,18-20]$. Similarly, only half of the providers in this study estimated that $75 \%$ or more of their Asian patients were screened for HBV infection and less than half of the providers had vaccinated the majority of their eligible Asian patients. Both patient and provider factors impact HBV screening and vaccination and provider knowledge represents an especially important factor $[14,17]$. Previous studies have shown that Asians are more likely to get screened and vaccinated against HBV if recommended by a medical provider [18]. In contrast, the lack of patient English-language fluency and low socioeconomic status are associated with lower rates of HBV screening and vaccination [21]. The majority of the patients in the practices surveyed in this study had low socioeconomic status and were uninsured or had public medical insurance and approximately half of the providers indicated that more than $50 \%$ of their Asian patients spoke little or no English. Our results, however, did not show an association between provider race (Asian vs non-Asian) or ability to speak an Asian Language and increased HBV or HCC screening. This finding may be due to the sample size, which may not be adequately powered to detect a difference. However, while one might expect that providers who are Asian or who speak an Asian language may have a heightened awareness of hepatitis B and better communicate the need for HBV and HCC screening to their patients, the data in the literature are conflicting. While there are reports of providers with Asian language abilities performing more HBV screening among Chinese-speaking patients [15], others have not found an association between provider race (Asian vs non-Asian) and Asian language and HCC screening among at-risk populations similar to our study [14]. In fact, in our study, only provider familiarity with HBV screening and vaccination recommendations rather than patient related factors were significantly associated with higher rates of HBV screening. Thus, providertargeted interventions should address ways to enhance their knowledge of current recommendations.

We have identified some deficit in knowledge with respect to HBV screening modalities utilized among providers. The combination of HBsAg and Anti-HBs has been recommended for HBV screening [22]. Nearly all providers in our study correctly included HBsAg, a test that detects active disease, as an HBV screening test, but $48 \%$ did not test for anti-HBs, which is necessary to assess eligibility for vaccination. This may reflect the lack of knowledge about the need for vaccination in this highrisk population. Moreover, this may lead to unnecessary 
vaccination of those already immune, thereby driving up costs in a resource-poor healthcare system.

The most common cause of HCC among Asian Americans is $\mathrm{CHB}$, and strategies for primary and secondary HCC prevention are critical in reducing cancer disparity in this group. In this study, we investigated provider knowledge of HCC and attitudes towards HCC screening. The majority of providers who responded were well informed about the risk of HCC among Asian Americans with CHB and recognized that primary prevention with HBV treatment can decrease the incidence of HCC [22-24]. Secondary prevention with HCC surveillance is recommended by national organizations. However, improvement in rates of HCC surveillance performed by this group of safety net healthcare providers is required in order to adhere to these recommendations.

There are limitations to the available data on survival benefit of HCC screening. Nevertheless, one randomized controlled trial and other prospective observational studies have shown that HCC screening may result in reduced mortality, specifically when curative therapy is available $[11,25,26]$. Similar to other survey studies, nearly all of the providers in this study agreed that cancer surveillance should be performed when there is strong evidence that mortality is reduced [14] and that they had recommended HCC surveillance to their patients. However, about $30 \%$ of providers were either unsure or did not believe that HCC screening in CHB patients improved survival and a third were unfamiliar with the AASLD guidelines endorsing surveillance.

In this study, predictors of adherence to the AASLD guidelines using ultrasound every 6-12 months for HCC surveillance in at-risk populations were assessed [12]. The most common screening modality, utilized by about twothirds of the respondents, was a combination of ultrasound and AFP at the frequency of 6-12 months. However, providers also used liver enzymes and HBV viral load, as well as radiologic modalities other than ultrasound that are not recommended as HCC screening tests. Factors associated with performing the recommended screening test in multivariable analysis included having a high proportion of at-risk Asian Americans in practice and greater provider knowledge of HBV-associated HCC. In a national survey of 459 primary providers and specialists identified through the American Medical Association Masterfile, Nguyen et al. [14] showed that the physicians who had a higher knowledge of HBV management were more likely to screen for $\mathrm{HCC}$ and that the concern for malpractice risk and the use of HCC screening as a quality measure increased the odds of screening. In another survey of 217 family physicians, HCC screening in CHB patients was related to years in practice, female physicians, and group practice [27]. In contrast, we did not find an independent association between HCC surveillance and physician characteristics or the overall provider attitude score, which included risk of malpractice and quality indicators.

The main limitation of this study is self-reported practice patterns, whereas the main strength is the high response rate. However, in general self-report tends to overestimate screening rates [28] and thus this limitation is unlikely to threaten our finding of suboptimal reported rates of screening for HBV and HCC by the study population. Generalizations to other practice settings is also limited, since surveyed practices had a high proportion of Asians, which may result in a heightened awareness of CHB and HCC disease that can underestimate the deficiencies in provider knowledge and rates of HBV and HCC screening in other practice settings with lower at-risk patient populations. Nevertheless, our findings uncovered suboptimal HBV screening and vaccination rates even among providers with high exposure to at-risk populations. As several factors including patient adherence likely play a role in rates of HBV screening, vaccination, and HCC surveillance, review of patient health records are required to confirm results obtained from provider reports.

In summary, our findings suggest that HBV screening and vaccination of eligible patients and education about the recommended tests for HBV screening and HCC surveillance are potential areas requiring improvement among providers. However, safety net providers are knowledgeable about cancer disparity among Asian Americans and are motivated to perform HCC surveillance even in the presence of limited resources. Our research supports the findings of the recent Institute of Medicine Report on Hepatitis and Liver Cancer [17] that ongoing education of patients and providers as well as investment in infrastructure to improve access to and quality of $\mathrm{HBV}$ care are key intervention strategies to reduce the HBV disease burden and the related cancer disparity among Asian Americans.

Acknowledgments This work was supported by National Institute of Health Grant numbers U01 CA114640-01S1 (M.K.) and P01 CA109091-01A1 (T.N., S.S., M.C.). Its contents are solely the responsibility of the authors and do not necessarily represent the official views of the NIH.

Open Access This article is distributed under the terms of the Creative Commons Attribution Noncommercial License which permits any noncommercial use, distribution, and reproduction in any medium, provided the original author(s) and source are credited.

\section{References}

1. Lavanchy D. Hepatitis B virus epidemiology, disease burden, treatment, and current and emerging prevention and control measures. J Viral Hepat. 2004;11:97-107.

2. El-Serag HB. Epidemiology of hepatocellular carcinoma in USA. Hepatol Res. 2007;37(Suppl 2):S88-S94. 
3. Miller BA, Chu KC, Hankey BF, Ries LA. Cancer incidence and mortality patterns among specific Asian and Pacific Islander populations in the U.S. Cancer Causes Control. 2008;19:227-256.

4. Wong R, Corley DA. Racial and ethnic variations in hepatocellular carcinoma incidence within the United States. Am J Med. 2008;121:525-531.

5. Lin SY, Chang ET, So SK. Why we should routinely screen Asian American adults for hepatitis B: a cross-sectional study of Asians in California. Hepatology. 2007;46:1034-1040.

6. Characteristics of persons with chronic hepatitis B-San Francisco, California, 2006. MMWR Morb Mortal Wkly Rep. 2007;56: 446-448.

7. Bindman AB, Chen A, Fraser JS, Yee HF Jr, Ofman D. Healthcare reform with a safety net: lessons from San Francisco. Am J Manag Care. 2009;15:747-750.

8. Chen MS Jr. Cancer health disparities among Asian Americans: what we do and what we need to do. Cancer. 2005;104:2895-2902.

9. Guy J, Yee HF Jr. Health disparities in liver disease: time to take notice and take action. Hepatology. 2009;50:309-313.

10. Ferenci P, Fried M, Labrecque D, et al. Hepatocellular carcinoma (HCC): a global perspective. J Clin Gastroenterol. 2010;44: 239-245.

11. Zhang BH, Yang BH, Tang ZY. Randomized controlled trial of screening for hepatocellular carcinoma. J Cancer Res Clin Oncol. 2004;130:417-422.

12. Bruix J, Sherman M. Management of hepatocellular carcinoma. Hepatology. 2005;42:1208-1236.

13. Chalasani N, Said A, Ness R, Hoen H, Lumeng L. Screening for hepatocellular carcinoma in patients with cirrhosis in the United States: results of a national survey. Am J Gastroenterol. 1999;94: 2224-2229.

14. Nguyen TT, Gildengorin G, Truong A, McPhee SJ. Factors influencing physicians' screening behavior for liver cancer among high-risk patients. J Gen Intern Med. 2007;22:523-526.

15. Lai CJ, Nguyen TT, Hwang J, Stewart SL, Kwan A, McPhee SJ. Provider knowledge and practice regarding hepatitis B screening in Chinese-speaking patients. J Cancer Educ. 2007;22:37-41.

16. Weinbaum CM, Mast EE, Ward JW. Recommendations for identification and public health management of persons with chronic hepatitis B virus infection. Hepatology. 2009;49:S35S44.

17. Mitchell AE, Colvin HM, Palmer Beasley R. Institute of Medicine recommendations for the prevention and control of hepatitis B and C. Hepatology;51:729-733.

18. Coronado GD, Taylor VM, Tu SP, et al. Correlates of hepatitis B testing among Chinese Americans. J Community Health. 2007;32: 379-390.

19. Ma GX, Shive SE, Wang MQ, Tan Y. Cancer screening behaviors and barriers in Asian Americans. Am J Health Behav. 2009;33: $650-660$.

20. Thompson MJ, Taylor VM, Jackson JC, et al. Hepatitis B knowledge and practices among Chinese American women in Seattle, Washington. J Cancer Educ. 2002;17:222-226.

21. Ma GX, Shive SE, Toubbeh JI, Tan Y, Wu D. Knowledge, attitudes, and behaviors of Chinese hepatitis B screening and vaccination. Am J Health Behav. 2008;32:178-187.

22. Lok AS, McMahon BJ. Chronic hepatitis B: update 2009. Hepatology. 2009;50:661-662.

23. Liaw YF, Sung JJ, Chow WC, et al. Lamivudine for patients with chronic hepatitis B and advanced liver disease. $N$ Engl J Med. 2004;351:1521-1531.

24. Lin SM, Sheen IS, Chien RN, Chu CM, Liaw YF. Long-term beneficial effect of interferon therapy in patients with chronic hepatitis B virus infection. Hepatology. 1999;29:971-975.

25. Chen JG, Parkin DM, Chen QG, et al. Screening for liver cancer: results of a randomised controlled trial in Qidong, China. J Med Screen. 2003;10:204-209.

26. McMahon BJ, Bulkow L, Harpster A, et al. Screening for hepatocellular carcinoma in Alaska natives infected with chronic hepatitis B: a 16-year population-based study. Hepatology. 2000; 32:842-846.

27. Ferrante JM, Winston DG, Chen PH, de la Torre AN. Family physicians' knowledge and screening of chronic hepatitis and liver cancer. Fam Med. 2008;40:345-351.

28. Rauscher GH, Johnson TP, Cho YI, Walk JA. Accuracy of selfreported cancer-screening histories: a meta-analysis. Cancer Epidemiol Biomarkers Prev. 2008;17:748-757. 